\title{
An Iterative Algorithm to Estimate LIDAR Ratio for Thin Cimus Cloud over Aerosol Layer
}

\author{
Zhenzhu Wang*, Dong Liu, Chenbo Xie, and Jun Zhou \\ Key Laboratory of Atmospheric Composition and Optical Radiation, Anhui Institute of Optics and Fine \\ Mechanics, Chinese Academy of Sciences, Hefei 230031, China
}

(Received May 12, 2011 : revised July 6, 2011 : accepted July 6, 2011)

\begin{abstract}
A new iterative algorithm is developed to estimate LIDAR ratio for a thin cirrus cloud over an aerosol layer. First, the thin cirrus cloud is screened out and replaced by a modeled LIDAR signal and the extinction coefficients of the aerosol layer are derived using the Fernald backward method. These aerosol coefficients are referred as the "actual values". Second, the original LIDAR signal which includes the thin cirrus cloud is also inverted by the Fernald backward method down to the aerosol layer but using different LIDAR ratio for the thin cirrus cloud. Depending on the different assumptions about the LIDAR ratio of the thin cirrus cloud, different sets of aerosol extinction can be derived. The "actual values" which are found in the first step can be used to constrain this iterative progress and the correct LIDAR ratio of the thin cirrus cloud can be found. The detailed description of this method and retrieval examples are given in the paper. The cases compared with other methods are presented and the statistical result is also shown and agrees well with other studies.
\end{abstract}

Keywords: LIDAR ratio, Thin cirrus cloud, Aerosol extinction, Iterative algorithms

OCIS codes : (010.3640) Lidar; (010.1615) Clouds; (280.1100) Aerosol detection

\section{INTRODUCTION}

Cirrus clouds are one of the most commonly occurring cloud types globally and they cover nearly $40 \%$ of the earth's surface as shown by earlier studies [1-2]. Cirrus clouds play an important role in the Earth's radiation budget because of their absorption of long-wave outgoing radiation from the Earth's surface and their reflection of incoming solar radiation. Therefore, knowledge of physical and optical properties of the cirrus clouds is essential for climate models. LIDAR offers an excellent way to obtain high accuracy cirrus data including height, shape and/or optical properties with high spatial and temporal resolution as shown by earlier studies [3-5]. As one of the most important parameters to investigate the optics of cirrus clouds, the LIDAR ratio for cirrus clouds, which is defined as the ratio of the cloud extinction and backscatter coefficients, has been attracting much attention. It can provide information on the transmission and reflection properties of cirrus clouds and also on the ice crystal properties due to its dependence on the size, shape, and orientation of the particles. And it varies with cloud types and varies even within any cloud because the cloud particle size distribution varies with height according to Sassen [6]. The availability of a reliable data set of LIDAR ratio for different cirrus types and conditions will greatly improve the quality of the cirrus properties derived from space, which will produce a global climatology of cirrus range resolved properties. Some variation of LIDAR ratio in the cloud layer can be measured by Raman LIDARs and high spectral resolution LIDARs according to Ansmann [7] and Grund [8], but there are still many ground-based elastic scattering LIDARs world-wide, through networks like NASA MPLNET or Asian Skynet, where determining the LIDAR ratio is very important. The average value of this ratio for the cloud layer is, however, a useful quantity, as it allows solutions for profile of extinction coefficient to be determined, especially for cirrus cloud layers. So far, a number of authors $[4,9-10]$ have reported LIDAR ratio retrieval methods for the cirrus clouds based on Mie LIDARs, and in this literature the cloud transmittance must be determined for the calculation of the LIDAR ratio. In order to give a better estimate of the transmittance than if the values being

\footnotetext{
*Corresponding author: zzwang@aiofm.ac.cn

Color versions of one or more of the figures in this paper are available online.
} 
used are only obtained at the heights of cloud base and top, a linear regression method according to Stuart [9] or a fitting method according to Chen [10] is used to match the LIDAR signal, but the uncertainty in the value of the transmittance will lead to a large relative error, especially when the optical depth of the cirrus cloud layer is small. In this paper, the authors introduce a new iterative algorithm to estimate LIDAR ratio for thin cirrus cloud based on solution of the Mie LIDAR equation without directly constraining the cloud transmittance.

The term thin cirrus cloud over an aerosol layer refers to a cloud that is visible or invisible, which can be penetrated by the LIDAR to detect a signal from the atmosphere with an aerosol layer below it. This kind of cloud generally has a small optical depth and lies at a high altitude without taking the multiple-scattering effect for granted as mentioned in reference [10]. The restriction may seem somewhat strict, but for our purposes it is quite a useful and practical one.

The algorithm methods for obtaining the LIDAR ratio for the cirrus cloud by inversion of Mie LIDAR signal are expressed in Section 2 and the results of applying these methods to measured cloud signals are shown in Section 3 with conclusions in the last section.

\section{METHODS}

In this section algorithms for the analysis of LIDAR signals containing the thin cirrus cloud layer are considered. The traditional Fernald-method according to Fernald [11] is introduced into the algorithm and applied to LIDAR signals reiteratively through guesses of LIDAR ratio for the cirrus cloud layer.

\subsection{Femald-method}

The LIDAR equation can be described as the following [11]:

$$
P(r)=\frac{K}{r^{2}} \cdot\left[\beta_{M}(r)+\beta_{P}(r)\right] \cdot T_{M}^{2}(r) \cdot T_{P}^{2}(r)
$$

Where $P(r)$ is the returned signal from atmosphere molecules and particles (e.g., aerosol and cloud) by backscattering at a range $r ; K$ is a system constant including output energy, receiver area, etc.; $\beta_{M}(r)$ and $\beta_{P}(r)$ are the molecular and particle backscatter coefficients at the range $r$, respectively. Note that

$$
T_{M}(r)=\exp \left(-\int_{0}^{r} \alpha_{M}(r) d r\right) \text { and } T_{P}(r)=\exp \left(-\int_{0}^{r} \alpha_{P}(r) d r\right)
$$

are the molecular and particle transmittance from the LIDAR to the range $r$, where $\alpha_{M}(r)$ and $\alpha_{P}(r)$ are the molecular and particle extinction coefficients, respectively.

The variable of range-square corrected signal $X(r)$ (i.e., $\left.P(r) \cdot r^{2}\right)$ is often used when solving the LIDAR equation for extinction as in the method by Fernald [11]. In the following, the two-component solution to the Eq. (1) for the particle extinction coefficient is given after knowing of the prior information at the calibration range $r_{\mathrm{c}}$.

$$
\begin{aligned}
\alpha_{P}(r)= & -\frac{S_{P}}{S_{M}} \cdot \alpha_{M}(r) \\
& +\frac{X(r) \cdot \exp \left[2\left(\frac{S_{P}}{S_{M}}-1\right) \cdot \int_{r}^{r_{c}} \alpha_{M}(r) d r\right]}{\frac{X\left(r_{c}\right)}{\alpha_{P}\left(r_{c}\right)+\frac{S_{P}}{S_{M}} \cdot \alpha_{M}\left(r_{c}\right)}+2 \int_{r}^{r_{c}} X(r) \cdot \exp \left[2\left(\frac{S_{P}}{S_{M}}-1\right) \cdot \int_{r}^{r_{c}} \alpha_{M}(r) d r\right] d r}
\end{aligned}
$$

Where $S_{M}=\alpha_{M}(r) / \beta_{M}(r)=8 \pi / 3$ is the LIDAR ratio for atmosphere molecular and $S_{P}=\alpha_{P}(r) / \beta_{P}(r)$ is the one for particles.

Note that here the particles can be aerosol (A: subscript abbr.) and/or cloud (C: subscript abbr.) below.

\subsection{Cloud-screen Method}

To find a cloud layer in a LIDAR signal and determine the ranges of its base and top has been discussed by many authors $[3,5,9,12-15]$. The methods use various algorithms that search for a zero crossing in the derivation of the signal or look for a threshold value as a criterion of cloud signal or construct a referenced LIDAR signal to test the presence of cloud, etc. All of those mentioned above are considered the spatial changes of LIDAR signals among various targets such as clouds and aerosols and are very useful under different conditions. For the types of thin cirrus cloud considered in this paper, a simple cloud detection algorithm based on high temporal resolution of observations has been used to separate cloud from noise and aerosol signals. The method is described in detail in the literature according to Wang [16] and will be summarized here.

For each measurement, a set of time-series signal profiles are obtained and used for finding the cloud layer by comparing the signal variations. The standard deviation at each range for the average signal profile is calculated and used for detecting special layers according to obvious signal variations with time. Then the ratio of the standard deviation profile to the averaged signal profile is considered to distinguish the cloud from the aerosol layer. The selection of the criteria for screening out the cirrus cloud layer is produced after an initial examination of the LIDAR data. Also, smoothing of the LIDAR signal is needed to promote data quality through applying sliding five-point linear least-square fitting to the noisy LIDAR signals from the thin cirrus clouds and aerosols.

\subsection{Iterative Algorithm - LIDAR Ratio Determined Method}

In order to derive the LIDAR ratio for a thin cirrus cloud over an aerosol layer, the iteration Fernald backward integrated method is used in analysis of a LIDAR signal. The solution is constrained by the values of extinction coefficients of the aerosol layer. A method expressed in the literature according to Wang [16] is used to determine aerosol properties under 
the cirrus-layer. The method can provide a better result than the traditional one because the influence of cloud layer on LIDAR signal inversion is eliminated. Here, a modeled LIDAR signal created by the local atmosphere molecular and aerosol model is used to replace the measured LIDAR signal from the range of base to top in the cirrus cloud. The extinction coefficients for the aerosol layer (hereafter, will be called "actual values") are retrieved by the Fernald backward integrated method based on this modified LIDAR signal and the inversion error due to substitution is less than $10 \%[16]$. Note here that the Eq. (2) is used with a constant assumption of LIDAR ratio for aerosol, which is $50 \mathrm{sr}$ at $532 \mathrm{~nm}$ wavelength.

The original LIDAR signal with thin cirrus cloud is also inverted by Fernald backward method down to the aerosol layer but using a different LIDAR ratio for thin cirrus cloud. According to guesses of LIDAR ratio for the thin cirrus cloud, a set of aerosol extinction coefficients (hereafter, will be called "estimate values" corresponded to "actual values") can be derived. The "actual values" can be used to constrain this iterative progress and the correct LIDAR ratio of the thin cirrus cloud can be estimated.

In fact, the choosing of the "actual values" covers some certain ranges below the thin cirrus cloud rather than the entire ranges from the LIDAR to the cloud base. Here, these ranges should be near but not touching the cloud base because the inverted results just below the cloud base are influenced by the cloud layer and the results down to the end far from the cloud layer converge so much that it is not easy to find correct LIDAR ratio through iteration. So, the ranges among the 500 1000 $\mathrm{m}$ below the cirrus layer are chosen generally.

And in the analysis software, the variables $S_{C g}, \alpha_{A e}(r)$ and $\alpha_{A a}(r)$ are defined to represent the guess of LIDAR ratio for the cloud, the "estimate values" and the "actual values" of aerosol extinction coefficient, respectively. The iterative process is controlled by the following condition in Eq. (3) through varying the LIDAR ratio $S_{C g}$.

$$
\left[\frac{1}{j-i+1} \cdot \sum_{r=r_{i}}^{r_{j}} \frac{\sqrt{\left(\alpha_{A e}(r)-\alpha_{A a}(r)\right)^{2}}}{\left|\alpha_{A a}(r)\right|}\right] \leq \frac{X}{100}
$$

Where $r_{i}$ and $r_{j}$ are the beginning and ending of the aerosol layer covered ranges with the bin number of $i$ and $j$ in the profile; and $X$ is the iteration criterion value, which is selected to maximize the agreement between the "estimate values" and the "actual values" but not to run into an unending loop. Here, the value $X=1$ is used suitably.

Note that here the evaluation for the variables is from 10 to $50 \mathrm{sr}$ according to the results of LIDAR ratio for cirrus clouds reported in the literature by Chen [4] and Xue [10]. The bisection method [17] is considered in the iteration so as to make the analysis progress more quickly than the usual step-iterative method. In the beginning of iteration, two sets of "estimate values" are computed by setting the value 10 and $50 \mathrm{sr}$ for $S_{C g}$. In the meantime, the signs of the value for $\alpha_{A e}(r)-\alpha_{A a}(r)$ inside Eq. (3) are also recorded, which are same in each setting for $S_{C g}$. If the signs are different, then the value 30 sr (i.e., mean value of 10 and $50 \mathrm{sr}$ ) for $S_{C g}$ is set to calculate new "estimate values". And the loop is continued through judgment of signs until the Eq. (3) is satisfied. The last guess of the LIDAR ratio for the cloud is the correct one.

\section{RESULTS}

The above methods have been used for analyzing cloud and aerosol LIDAR data at $532 \mathrm{~nm}$ wavelength. The data has been observed over Hefei by PML (Polarization-Mie LIDAR) by Wang [18-19]. An example of application of the iterative algorithm is given below; along with a case of the analysis in an extension of the analysis to a situation when the cirrus cloud layer passes intermittently through the detection area. And the results compared with other methods are also considered and analyzed in the following.

\subsection{Case I}

The LIDAR signal shown in Fig. 1(a) was observed on Mar. 9th of 2009 with a thin cirrus cloud over an aerosol

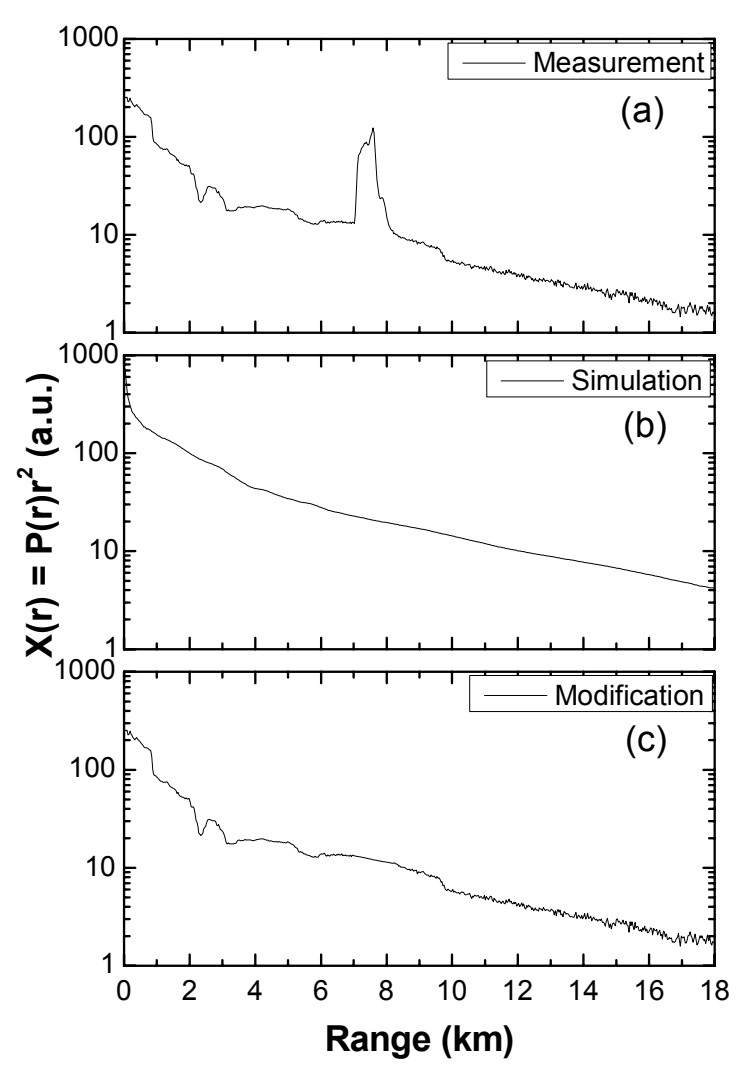

FIG. 1. Case I: (a) Measured LIDAR signal from cirrus cloud and aerosol. (b) Simulated LIDAR signal with atmosphere molecular and aerosol model data. (c) Modified LIDAR signal based on the measured signal and the simulated one. 


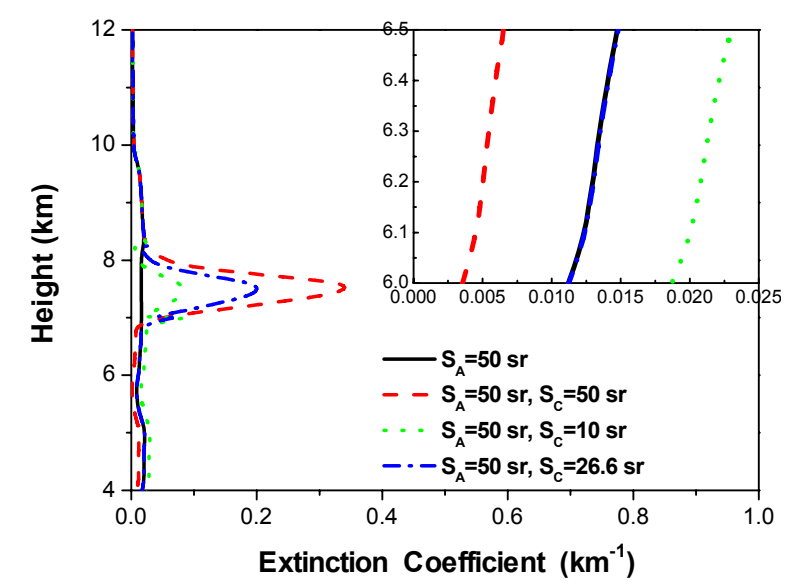

FIG. 2. Retrieved extinction coefficient profiles using Fernald method from the modified signal (the solid line) and that from the original measured signal (the dashed, dotted and dash-dotted lines) in Case I.

layer. The cloud layer, whose height is $7.02 \mathrm{~km} \sim 8.22 \mathrm{~km}$ with a peak height $7.59 \mathrm{~km}$, is screened out by the method mentioned in the literature by Wang [16]. The simulated LIDAR signal plotted in Fig. 1(b) is created by suitable molecular and aerosol model data in addition to the LIDAR system parameters. Note that here the molecular data is derived from U. S. Standard Atmospheric Model and Rayleigh scattering theory; the aerosol data is averaged and fitted using the routine measurements of troposphere aerosols obtained in cloudless and fair nights during 1998-2008 by a $532 \mathrm{~nm}$ Mie LIDAR over Hefei. For the profile in Fig. 1(b), only spring data are used because the measurement in Fig. 1(a) is carried out in this season. By using the simulated signal to match the measured one at the ranges of the cloud layer from 7.02 to $8.22 \mathrm{~km}$, a modified one is arrived at shown in Fig. 1(c).

Here, the signal above the cirrus cloud is corrected through considering the transmittances of simulated aerosol layer and cloud layer, where that of simulated aerosol layer (The value is 0.9100 .) can be computed directly using the signals at 7.02 and $8.22 \mathrm{~km}$ and that of cloud layer (The value is 0.8732 .) is calculated by Chen's [4] fitting method.

The profiles for aerosol and/or cirrus cloud extinctions in Fig. 2 are calculated using the Fernald method with the same calibrated range $r_{c}=14.79 \mathrm{~km}$, which is selected according to some criteria as shown by earlier studies [20-21] in analysis software. So, the retrieved results below $r_{c}$ are all inverted by backward integrated method. The solid line in the Fig. 2 is derived from the modified signal with an assumption of LIDAR ratio for aerosol $S_{A}=50 \mathrm{sr}$. Wang etc. have shown how to get a better aerosol extinction coefficient below the cirrus cloud and error analysis has been given [16]. We assume that this LIDAR ratio $\left(S_{A}=50\right)$ as the true one to restrain the iterative process. Another three lines (dashed, dotted and dash-dotted) in the same figure are derived from the original measured signal with the same

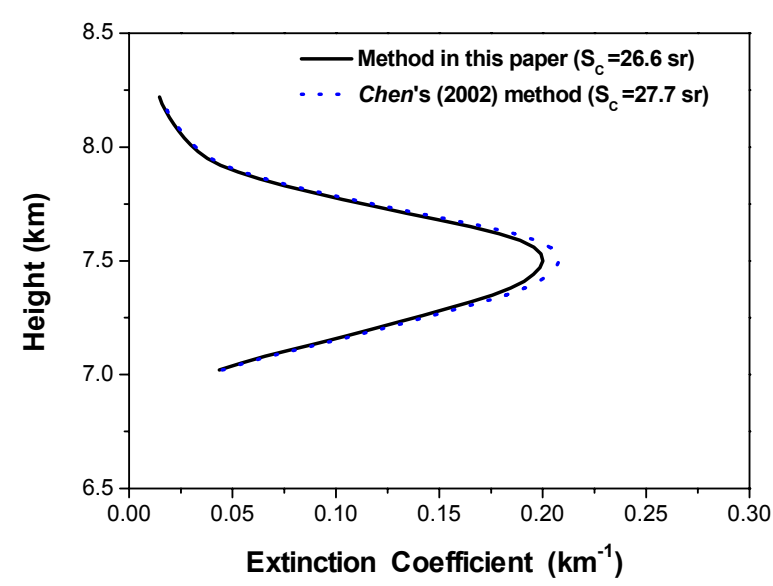

FIG. 3. Profiles of cirrus extinction coefficients derived from different LIDAR ratios estimated by our method (the solid line) and Chen's one (the dotted line) in Case I.

assumption of LIDAR ratio for aerosol and different guesses of LIDAR ratio for cirrus cloud $S_{C}=50,10$ and $26.6 \mathrm{sr}$, respectively. Note that here the first two LIDAR ratios are initial values and the last one is the correct one through iterative analysis, in which sign comparison (same or not) and criteria judgment (satisfied to Eq. 3 or not) are included. As shown in an enlargement in the inset of Fig. 2 for the reference ranges from 6 to $6.5 \mathrm{~km}$, the initial "estimate values" are far away from the "actual values" and the last "estimate values" are almost close to the "actual values".

In order to prove the method mentioned in this paper, the derived LIDAR ratio for the thin cirrus cloud is compared with that by Chen's [4] method, which is constrained by transmittance of the cloud. The results are $26.6 \mathrm{sr}$ and 27.7 sr respectively shown in Fig. 3, along with the profiles of cirrus extinction coefficients using the two LIDAR ratios. From the good agreement of these profiles, the method in the paper is reliable and practical.

\subsection{Case II}

Sometimes, there are not enough extinction profiles of aerosols for an averaged model data in some regions, so that a suitable simulated LIDAR signal cannot be obtained to continue the analysis process normally. In the following condition, the method to estimate LIDAR ratio for a thin cirrus cloud will be extended. to the case when a cirrus cloud layer passes through the laser path of LIDAR intermittently in a period of time.

Fig. 4 shows the measured LIDAR signals with a cloud layer over the LIDAR and a cloud free condition, which are obtained by PML on the afternoon of Mar. 7th, 2005. Also the cloud layer is screened out by Wang's [16] method with a boundary range from 10.35 to $11.07 \mathrm{~km}$, whose transmittance is 0.9762 calculated by Xue's [10] method. Note that here Chen's [4] method is not considered because some assumptions used in this method make it not adaptable when the transmittance of the cloud is more than 0.9700 


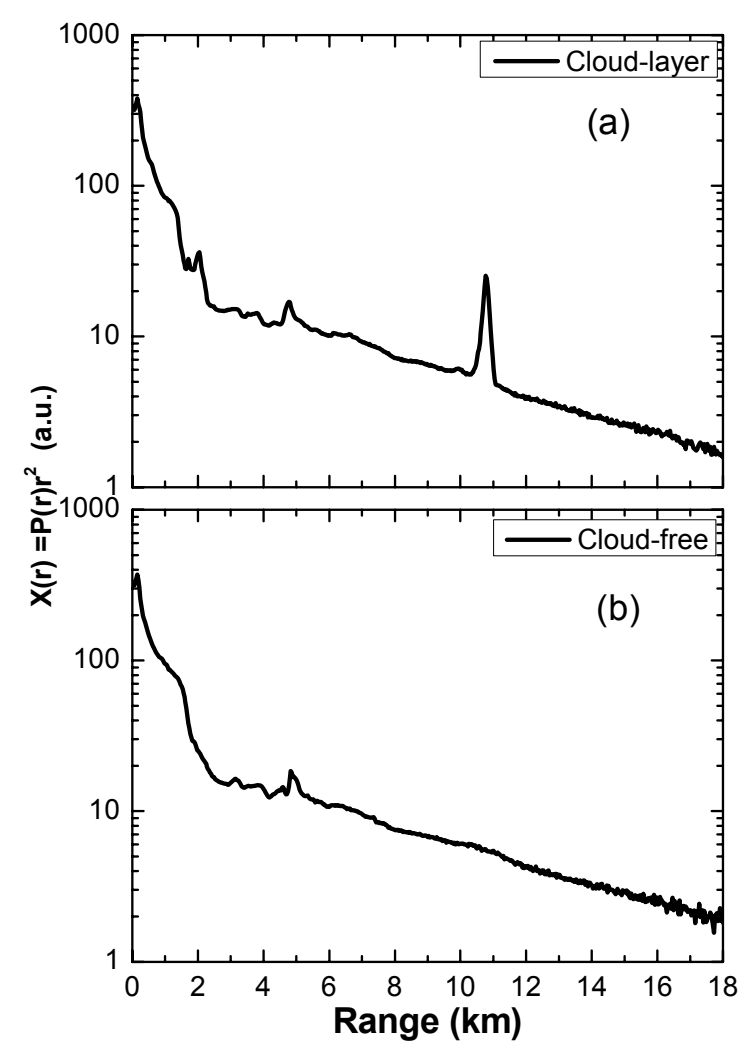

FIG. 4. Case II: Measured LIDAR signals in the conditions with (a) and without (b) the cirrus cloud layer.

and in this condition Xue's [10] method is better.

The profiles for aerosol and/or cirrus cloud extinctions in Fig. 5 are calculated using the Fernald method with a same calibrated range $r_{c}=11.97 \mathrm{~km}$ and also backward integration below it. The solid line in the figure 5 is derived from the cloud-free LIDAR signal with an assumption of LIDAR ratio for aerosol $S_{A}=50 \mathrm{sr}$. And another three lines (dashed, dotted and dash-dotted) in the same figure are derived from the cloud-passing LIDAR signal with the same assumption of LIDAR ratio for aerosol and different guesses of LIDAR ratio for cirrus cloud $S_{C}=50,10$ and $47.5 \mathrm{sr}$, respectively. Note that here the first two LIDAR ratios are also initial values and the last one is the correct one through iterative analysis as before. As shown in an enlargement in the inset of Fig. 5 among the reference ranges from 9.0 to $9.5 \mathrm{~km}$, the initial "estimate values" are apart from the "actual values" and the last "estimate values" are very close to the "actual values".

The derived LIDAR ratio for the thin cirrus cloud by our method is compared with that by Xue's [10] method, which is also constrained by transmittance of the cloud. The results are $47.5 \mathrm{sr}$ and $47.8 \mathrm{sr}$ respectively shown in Fig. 6, along with the profiles of cirrus extinction coefficients using the two LIDAR ratios. Their values agree with each other not only in LIDAR ratio but also in extinction coefficient and testify to the reliability of the method in this paper. But the limited use of this extension is obvious

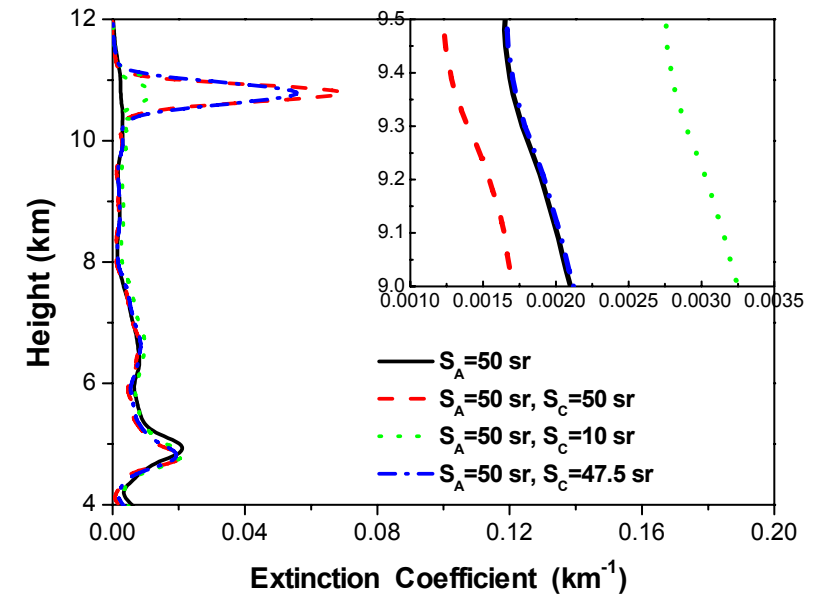

FIG. 5. Retrieved extinction coefficient profiles using Fernald method from the measured signal without cirrus cloud (the solid line) and that from the measured signal with cirrus cloud (the dashed, dotted and dash-dotted lines) in Case II.

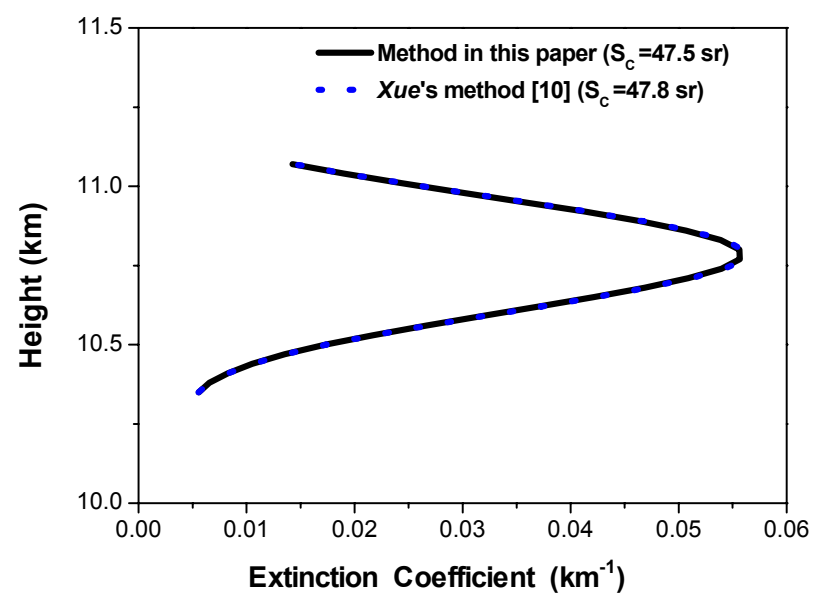

FIG. 6. Profiles of cirrus extinction coefficients derived from different LIDAR ratios estimated by our method (the solid line) and Xue's one (the dotted line) in Case II.

and it is only suitable for the conditions when the cloud lies intermittently over the LIDAR with a stable atmosphere condition below the cloud layer during the detecting period.

\subsection{Statistical Result}

The clouds measured by PML over Hefei $(31.897 \mathrm{~N}$, 117.173E) in spring (Mar., Apr., May) of 2005 2008 are analyzed. There are total 301 data samples produced from selected cirrus cloud sets. In order to ensure SNR for cloud data, one cloud profile is generated by about $50 \mathrm{~s}$ LIDAR measurement time and ten profiles are averaged to produce one LIDAR ratio for a corresponding cirrus clouds. The iterative method is employed here. The statistical results are shown in Fig. 7 and the LIDAR ratio for thin cirrus cloud varies from 5 to $65 \mathrm{sr}$ with a mean value 31.2 \pm 11.7 sr. The result can be compared with other studies. 


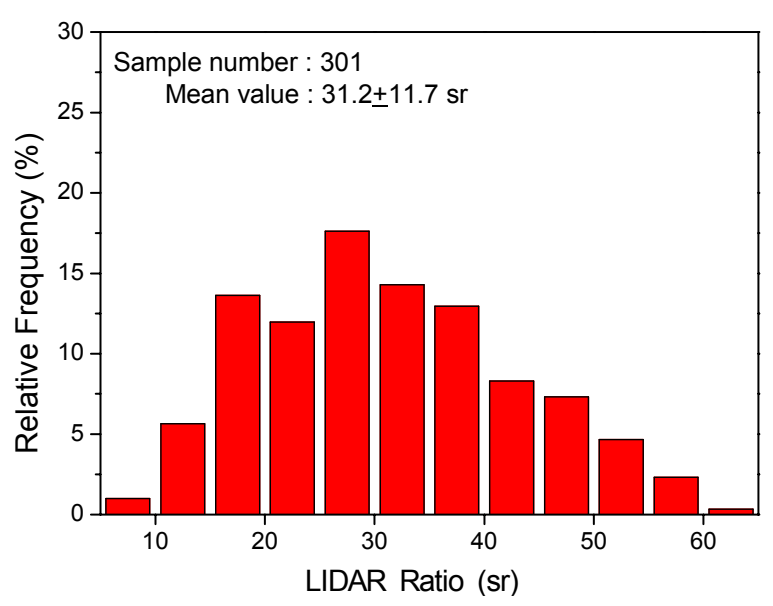

FIG. 7. Frequency distribution of LIDAR ratio for cirrus clouds observed by PML over Hefei in spring of 2005 2008.

Xue [22] reported LIDAR ratio about $30.6 \pm 9.4$ sr for high-altitude cirrus cloud over Hefei (31.897N, 117.173E). Chen [4] derived the inverse LIDAR ratio with a mean value $29 \pm 12$ sr for cirrus cloud over Chung-li $(25 \mathrm{~N}$, 121E). Their values agree well with our study.

\section{CONCLUSIONS}

A new iterative algorithm is developed to estimate LIDAR ratio for thin cirrus cloud over an aerosol layer, in which the Fernald backward method is considered by using different LIDAR ratio for thin cirrus cloud to satisfy the criteria in our analysis software. The values of aerosol extinction below the cloud layer are used to control the iterative process other than the transmittance of the cloud, which is always over- or under- estimated due to the complexity of cloud and will induce bias error. The algorithm is also extended to the conditions in which the cloud lies intermittently within the detection area. Two applied cases are discussed and compared with other methods and good agreement is obtained. The statistical LIDAR ratio for thin cirrus cloud over Hefei in spring of 2005 2008 averaged about $31.2 \mathrm{sr}$ and shows a good agreement with other studies.

With this method, the best results are obtained when there is only one cloud layer. Obviously, the use of the same LIDAR ratio for several different cloud layers contained in one LIDAR signal is of limited physical use. Note, however, that if the cloud layers are sufficiently separated in height to allow the choosing of an aerosol layer below each cloud layer, then our method can estimate LIDAR ratio for each layer one by one.

\section{ACKNOWLEDGMNT}

This work was supported by the National Key Basic Research
Project of China under Grant by No.2007FY110700 and the National Basic Research Program of China under Grant No.2011CB403401. The authors wish to thank two anonymous reviewers and Prof. Z. M. Tao for useful comments and suggestions.

\section{REFERENCES}

1. H. Nazaryan, M. P. McCormick, and W. P. Menzel, "Global characterization of cirrus clouds using CALIPSO data," J. Geophys. Res. 113, D16211 (2008).

2. K. Sassen, Z. Wang, and D. Liu, "Global distribution of cirrus clouds from cloudsat/cloud-aerosol LIDAR and infrared pathfinder satellite observations (CALIPSO) measurements," J. Geophys. Res. 113, D00A12 (2008).

3. K. Sassen and B. S. Cho, "Subvisual-thin cirrus LIDAR dataset for satellite verification and climatological research," J. Appl. Meteor. 31, 1275-1285 (1992).

4. W. Chen, C. Chiang, and J. Nee, "LIDAR ratio and depolarization ratio for cirrus clouds," Appl. Opt. 41, 6470-6476 (2002).

5. Z. Wang and K. Sassen, "Cloud type and macrophysical property retrieval using multiple remote sensors," J. Appl. Meteor. 40, 1665-1682 (2001).

6. K. Sassen and R. L. Petrilla, "LIDAR depolarization and multiple scattering in marine stratus clouds," Appl. Opt. 25, 1450-1459 (1986).

7. A. Ansmann, U. Wandinger, M. Riebesell, C. Weitkamp, and W. Michaelis, "Independent measurement of extinction and backscatter profiles in cirrus clouds using a combined Raman elastic-backscatter LIDAR," Appl. Opt. 31, 7113-7131 (1992).

8. C. J. Grund and E. W. Eloranta, "The 27-28 October 1986 FIRE IFO cirrus case study: cloud optical properties determined by high spectral resolution LIDAR," Mon. Weather Rev. 118, 2344-2355 (1990).

9. A. Y. Stuart, "Analysis of LIDAR backscatter profiles in optically thin cloud," Appl. Opt. 34, 7019-7030 (1995).

10. X. Xue and J. Zhou, "A method to determine averaged LIDAR ratio in high-altitude thin cirrus," Chinese Journal of Quantum Electronics 23, 115-119 (2006).

11. F. G. Fernald, "Analysis of atmospheric LIDAR observation: some comments," Appl. Opt. 23, 652-653 (1984).

12. R. P. Shiv, W. Steinbrecht, and I. C. Allan, "Automated method for LIDAR determination of cloud-base height and vertical extent," Appl. Opt. 31, 1488-1494 (1992).

13. D. M. Winker and M. A. Vaughan, "Vertical distribution of clouds over Hampton, Virginia, observed by LIDAR under the ECLIPS and FIRE ETO programs," Atmos. Res. 34, 117-133 (1994).

14. K. Sassen and Z. Wang, "The need for a universal cloud property algorithm for active remote sensors," in Proc. Ninth Atmospheric Radiation Measurement (ARM) Science Team Meeting (San Antonio, TX, USA, 1999), pp. 169-173.

15. J. R. Campbell, D. L. Hlavka, J. D. Spinhirne, D. D. Turner, and C. J. Flynn, "Operational cloud boundary detection and analysis from micro pulse LIDAR data," in Proc. Eighth Atmospheric Radiation Measurement (ARM) Science Team Meeting (Tucson, AZ, USA, 1998), pp. 119-122. 
16. Z. Wang, D. Wu, D. Liu, and J. Zhou, "An algorithm to determine aerosol properties by eliminating cirrus-layer influence on Mie-LIDAR signals,” J. Opt. Soc. Korea 14, 444-450 (2010).

17. R. P. Brent, Algorithms for Minimization without Derivatives (Prentice-Hall, Englewood Cliffs, NJ, USA, 1973), Chapter 4.

18. Z. Wang, R. Chi, B. Liu, and J. Zhou, "Depolarization properties of cirrus clouds from polarization LIDAR measurements over Hefei in spring," Chinese Optics Letters 6, 235-237 (2008).

19. Z. Wang, D. Liu, J. Zhou, and Y. Wang, "Experimental determination of the calibration factor of polarization-Mie LIDAR,” Opt. Rev. 16, 566-570 (2009).
20. Y. Sasano, "Tropospheric aerosol extinction coefficient profiles derived from scanning LIDAR measurements over Tsukuba, Japan, from 1990 to 1993," Appl. Opt. 35, $4941-4952$ (1996).

21. J. Zhou, G. Yue, C. Jin, F. Qi, D. Liu, H. Hu, Z. Gong, G. Shi, T. Nakajima, and T. Takamura, "LIDAR observation of Asian dust over Hefei, China, in spring 2000," J. Geophys. Res. 107, 4252-4259 (2002).

22. X. Xue and J. Zhou, "LIDAR observations of cirrus clouds over Hefei," Chinese Journal of Quantum Electronics 23, 527-532 (2006). 